\title{
Neues Konjunkturrisiko: Engpässe bei Materialien und Vorprodukten
}

In den vergangenen Monaten sind Lieferengpässe bei Vorprodukten wie Halbleitern und Materialien wie Stahl, Holz und Kunststoffen zu einem wichtigen Belastungsfaktor für die Wirtschaftsentwicklung geworden. Im August 2021 berichteten in ifoUnternehmensbefragungen $70 \%$ der Unternehmen des Verarbeitenden Gewerbes, dass derartige Engpässe ihre Produktion behindern. Das ist ein historischer Rekordwert. In früheren Aufschwungphasen hat es zwar immer wieder Beschaffungsprobleme gegeben, aber der Anteil der betroffenen Industriefirmen hat in den letzten drei Jahrzehnten maximal $20 \%$ erreicht. Die aktuellen Friktionen sind also deutlich gravierender als das, was wir aus der jüngeren Vergangenheit kennen.

Am stärksten betroffen ist die Autoindustrie, wo 91,5\% der Firmen über Engpässe klagen. Beim Maschinenbau sind es mehr als $80 \%$, in der Chemischen Industrie $57 \%$. Unter den Branchen des Verarbeitenden Gewerbes ist die Getränkeindustrie am wenigsten eingeschränkt, aber auch dort trifft es beinahe ein Drittel der Unternehmen. Lieferengpässe gibt es auch außerhalb des Verarbeitenden Gewerbes, vor allem im Bausektor. Dort berichteten im August $38 \%$ der Firmen von Materialmangel.

Um welche Produkte geht es? Die Probleme bei der Lieferung von Halbleitern werden bereits seit längerer Zeit diskutiert. Der pandemiebedingte Digitalisierungsschub hat die Nachfrage nach Bauteilen für Computer besonders stark steigen lassen. Aber die Engpässe reichen weit darüber hinaus. Lieferschwierigkeiten gibt es bei Stahl, Kupfer und Aluminium, bei Holz, elektronischen Bauteilen, Kunststoffen, Verpackungsmaterialien und vielem mehr. Entsprechend groß ist die Zahl der Betroffenen.

Die Ursachen sind vielfältig. Nach dem Ausbruch der Corona-Krise 2020 haben viele Unternehmen die Investitionen drastisch gesenkt und Produktionskapazitäten reduziert. Vor allem die Industrieproduktion hat sich dann weltweit unerwartet schnell erholt. 2021 hat die Kombination aus umfangreichen staatlichen Konjunkturprogrammen, zurückgestauter Kaufkraft und zunehmend verfügbaren Impfungen die Erholung der Weltwirtschaft weiter beflügelt und die Nachfrage nach Vorprodukten und Materialien deutlich stärker als erwartet befeuert.

Eine kräftige Nachfrage, die inzwischen teils das Vorkrisenniveau überstiegen hat, traf auf verringerte Produktionskapazitäten und Förderkapazitäten bei Rohstoffen. Bei einzelnen Produkten wie Schnittholz, kamen Sondereffekte wie Produktionsausfälle aufgrund von Naturereignissen wie z. B. Käferbefall hinzu. Außerdem führten Handelsstreitigkeiten zwischen den USA und Kanada dazu, dass die Nachfrage nach europäischem Schnittholz in die Höhe schnellte.

Hinzu kommt, dass die Pandemie die gesamte Choreografie des globalen Gütertransports mit Containerschiffen von einem zum anderen Kontinent durcheinandergebracht hat und weiterhin stört. Zudem ist die Nachfrage zwar stark gestiegen, aber nicht synchron in allen Sektoren und Weltregionen, denn das Tempo der wirtschaftlichen Erholung ist sehr ungleich verteilt. Containerkapazitäten sind vorhanden, aber oft nicht dort, wo sie gebraucht werden. Aufgrund von Quarantäneverordnungen fallen vor allem in

(C) Der/die Autor:in 2021. Open Access: Dieser Artikel wird unter der Creative Commons Namensnennung 4.0 International Lizenz veröffentlicht (creativecommons.org/licenses/by/4.0/deed.de).

Open Access wird durch die ZBW - Leibniz-Informationszentrum Wirtschaft gefördert.

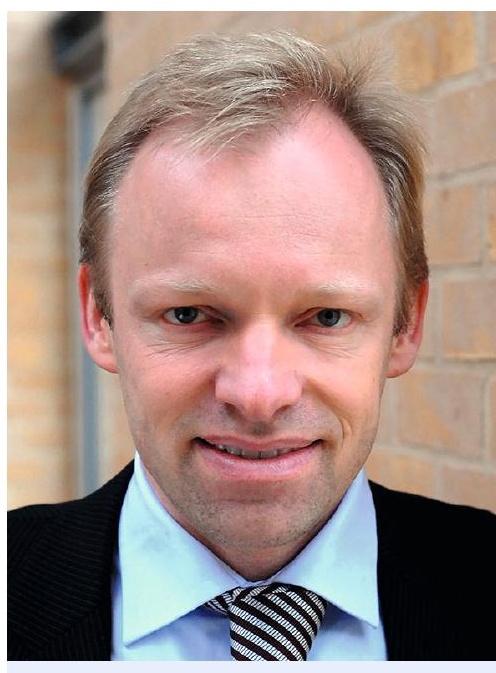

Clemens Fuest ist Präsident des ifo Instituts - Leibniz-Institut für Wirtschaftsforschung an der Universität München e.V. 
Asien, speziell in China, immer wieder Produktionsanlagen oder große Häfen vorübergehend aus. All das verschärft die Logistikprobleme und hat die Preise für Gütertransporte empfindlich steigen lassen. Gleichzeitig versuchen viele Unternehmen gerade wegen der anhaltenden Unsicherheit über weitere pandemiebedingte Störungen Lagerbestände mit Vorprodukten aufzubauen. Das verschärft die Güterknappheit zusätzlich.

Wie gehen die Unternehmen mit den aktuellen Engpässen um? Die Nachfrage nach Produkten des Verarbeitenden Gewerbes ist derzeit sehr gut; die Auftragsbücher sind für mehrere Monate gefüllt. Umso schmerzlicher ist es, dass fehlende Vorprodukte die Produktion hemmen. In den letzten Monaten haben viele Unternehmen Lager sowohl bei Vorprodukten als auch bei ihren eigenen Fertigwaren abgebaut. Aber diese Reserven sind jetzt größtenteils verbraucht - viele Aufträge können nicht mehr bedient werden.

Einzelne Unternehmen helfen sich, indem sie für knappe Vorprodukte sehr viel Geld bieten. Auch auf ihren Absatzmärkten erhöhen sie die Preise. Das ist durchaus sinnvoll, weil so Ressourcen in Bereiche gelenkt werden, in denen die Zahlungsbereitschaft besonders hoch ist. Allerdings kann diese Form der Anpassung kurzfristig, bei gegebener Menge verfügbarer Vorprodukte, nicht verhindern, dass die gesamtwirtschaftliche Produktion zurückgehalten wird. Gleichzeitig stellt sich die Frage, ob die Lieferengpässe einen Inflationsschub nach sich ziehen. Vorübergehend wird die Inflation sicherlich in die Höhe getrieben. Mittelfristig kann man aber davon ausgehen, dass die Lieferengpässe entschärft werden und damit auch der Effekt auf die Preisentwicklung nachlässt.

Welche Lehren sollten die Unternehmen aus den aktuellen Lieferproblemen ziehen, und was sind die Folgen für die Wirtschaftspolitik? Häufig wird gefordert, angesichts der Störanfälligkeit der globalen Lieferketten sollten Unternehmen mehr Komponenten selbst herstellen, mehr auf heimische Lieferanten setzen und höhere Lagerbestände vorhalten. Zweifellos werden viele Firmen zwischen Kostenvorteilen und Verlässlichkeit, Just-in-time-Produktion und Risikominderung durch Lagerhaltung neu abwägen. Mehr Resilienz der Produktion kann allerdings auch bedeuten, Risiken zu differenzieren, indem man bei wichtigen Vorprodukten auf eine größere Zahl von Lieferanten setzt. Aktuelle Erhebungen des ifo Instituts zeigen, dass viele Unternehmen diesen Weg gehen. Wirtschaftspolitisch ist die Forderung verbreitet, die Fertigung wichtiger Produkte, die bislang importiert werden, im Inland oder zumindest innerhalb der EU zu fördern. Das mag vertretbar sein bei einzelnen Produkten grundlegender Bedeutung, z. B. bei lebenswichtigen Medikamenten. Als allgemeine wirtschaftspolitische Strategie ist dieser Ansatz jedoch verfehlt, da er leicht in Protektionismus umschlagen kann. Gerade Deutschland mit seiner exportorientierten Wirtschaft sollte dem entgegentreten.

Was bedeutet das für die Konjunkturentwicklung? Viel wird davon abhängen, wie lange die Engpässe anhalten. Als die Lieferprobleme im Frühjahr 2021 deutlich wurden, haben viele gehofft, dass sich die Lage im Sommer entspannt, da Produktion und Logistik angesichts der großen Knappheit und stark steigender Preise ihre Leistung ausweiten. Dazu kommt es sicherlich, aber bislang ist noch keine Entspannung sichtbar, zumindest nicht bei den Unternehmen in Deutschland. Im Verarbeitenden Gewerbe hat sich die Lage im August gegenüber den Vormonaten noch einmal verschärft. Im Bausektor ist der Anteil der betroffenen Unternehmen zwar von seinem Höchststand von $46 \%$ im Juni auf $38 \%$ im August gesunken, aber diese Zahl ist immer noch sehr hoch. Da die Pandemie durch die Impfungen zwar deutlich entschärft, aber noch nicht überwunden ist, kann es auch weiterhin zu Produktionsausfällen und Verzögerungen beim Gütertransport kommen. Deshalb ist zu befürchten, dass die Engpässe sich bis 2022 hinziehen. Bislang gin-

Clemens Fuest gen die meisten Konjunkturprognosen davon aus, dass die deutsche Wirtschaft gegen

ifo Institut Ende 2021 das Vorkrisenniveau wieder erreicht. Angesichts der anhaltenden Lieferprofuest@ifo.de 